\title{
EDITORIAL
}

\section{Revisited Pulmonary Hypertension}

Pulmonary hypertension (PH) is a pathophysiological disorder that may involve multiple clinical conditions and can complicate the majority of cardiovascular and respiratory diseases. $\mathrm{PH}$ is defined as an increase in mean pulmonary arterial pressure (PAPm) $\leq 25 \mathrm{mmHg}$ at rest as assessed by right heart catheterization (RHC). Available data have shown that the normal PAPm at rest is $14+3 \mathrm{mmHg}$ with an upper limit of normal of approximately $20 \mathrm{mmHg}$. The clinical significance of a PAPm between 21 and $24 \mathrm{mmHg}$ is unclear. Haemodynamically pulmonary hypertension defined as pre and post-capillary PH. For all clinical groups Pulmonary Wedge Pressure ( PWP) is the most important component to classify Pre and Post-capillary PH. To define Pre-capillary PH, PWP should remain $<15 \mathrm{mmHg}$ as seen in Pulmonary arterial hypertension, $\mathrm{PH}$ due to lung diseases, Chronic thromboembolic PH ( CTEPH), PH with unclear and/or multifactorial mechanisms. In Post-capillary PH due to left heart disease PWP is usually $>15 \mathrm{mmHg}$.

Recently identified gene mutations have been included in the HPAH subgroup of clinical group 1 (PAH). The new mutations are more rare as compared with the traditional bone morphogenetic protein receptor 2 (BMPR2) mutations. Pre-capillary PH associated with chronic haemolytic anaemia appears to be significantly different from other forms of PAH.

An electrocardiogram (ECG) may provide supportive evidence of PH, but a normal ECG does not exclude the diagnosis. An abnormal ECG is more likely in severe rather than mild PH. ECG abnormalities may include P pulmonale, right axis deviation, $\mathrm{RV}$ hypertrophy, $\mathrm{RV}$ strain, right bundle branch block, and QTc prolongation. While RV hypertrophy has insufficient sensitivity (55\%) and specificity (70\%) to be a screening tool, RV strain is more sensitive. Prolongation of the QRS complex and QTC suggest severe disease. In contrast to PH, ECG changes in ischaemia more commonly affect the lateral and inferior leads, and when present in the anterior chest leads are usually accompanied by a Q wave in V1 to V3, and rarely cause right axis deviation. Supraventricular arrhythmias may occur in advanced disease, in particular atrial flutter, but also atrial fibrillation, with a cumulative incidence in $25 \%$ of patients after 5 years. Atrial arrhythmias compromise $\mathrm{CO}$ and almost invariably lead to further clinical deterioration.
In $90 \%$ of patients with IPAH (Idiopathic Pulmonary Arterial Hypertension) the chest radiograph is abnormal at the time of diagnosis. Findings in patients with PAH include central pulmonary arterial dilatation, which contrasts with 'pruning' (loss) of the peripheral blood vessels. Right atrium (RA) and RV( Right Ventricle) enlargement may be seen in more advanced cases. A chest radiograph may assist in differential diagnosis of $\mathrm{PH}$ by showing signs suggesting lung disease or pulmonary venous congestion due to LHD (Left Heart Disease). As for ECG, a normal chest radiograph does not exclude PH. Pulmonary function tests and arterial blood gases identify the contribution of underlying airway or parenchymal lung disease.

Transthoracic echocardiography is used to image the effects of PH on the heart and estimate PAP from continuous wave Doppler measurements. Echocardiography should always be performed when $\mathrm{PH}$ is suspected and may be used to infer a diagnosis of $\mathrm{PH}$ in patients in whom multiple different echocardiographic measurements are consistent with this diagnosis. When treatment of PH itself is being considered, echocardiography alone is not sufficient to support a treatment decision and cardiac catheterization is required. Two-dimensional, Doppler and contrast examinations can be used to identify CHD. High pulmonary blood flow found on pulsed wave Doppler in the absence of a detectable shunt or significant dilatation of proximal PA despite only moderate PH may warrant transoesophageal examination with contrast or cardiac magnetic resonance (CMR) imaging to exclude sinus. In cases of suspicion of LV diastolic dysfunction, Doppler echocardiographic signs should be assessed even if their reliability is considered low. RHC should be considered when the diagnosis remains uncertain after non-invasive investigations. A ventilation/perfusion (V/Q) lung scan should be performed in patients with $\mathrm{PH}$ to look for CTEPH. A normal- or lowprobability V/Q scan effectively excludes CTEPH with a sensitivity of $90-100 \%$ and a specificity of $94-100 \%$; CT is preferred in many centres since it is more readily available. More recently, newer techniques such as three dimensional magnetic resonance (MR) perfusion mapping, have been demonstrated to be as sensitive as traditional perfusion scintigraphy in screening for CTEPH; High-resolution 
computed tomography, contrast-enhanced computed tomography, and pulmonary angiography CT imaging is a widely available tool that can provide important information on vascular, cardiac, parenchymal and mediastinal abnormalities.A segmental artery:bronchus ratio. 1 : 1 in three or four lobes has been reported to have high specificity for $\mathrm{PH}$.

Contrast-enhanced and unenhanced MR angiography have a potential in the study of the pulmonary vasculature in patients with $\mathrm{PH}$. Routine biochemistry, haematology and thyroid function tests are required in all patients, as well as a number of other specific blood tests. Liver function tests may be abnormal because of high hepatic venous pressure, liver disease and/or endothelin receptor antagonist (ERA) therapy. Serological testing is required to detect underlying CTD, hepatitis and human immunodeficiency virus (HIV). Up to $40 \%$ of patients with IPAH have elevated antinuclear antibodies usually in a low titre (1:80). It is important to look for evidence of SSc since this disease has a relatively high prevalence of PAH. Limited scleroderma typically has antinuclear antibodies, including anti-centromere, dsDNA, anti-Ro, U3-RNP, B23, Th/To and U1-RNP. Patients with systemic lupus erythematosus may have anticardiolipin antibodies. Patients with CTEPH should undergo thrombophilia screening, including antiphospholipid antibodies, anticardiolipin antibodies and lupus anticoagulant. HIV testing is required in $\mathrm{PAH}$. $\mathrm{N}$-terminal pro-brain natriuretic peptide (NT-proBNP) may be elevated in patients with $\mathrm{PH}$ and is an independent risk predictor in these patients.
Abdominal ultrasound may confirm but not formally exclude portal hypertension. The use of contrast agents and the addition of a colour Doppler examination may improve the accuracy of the diagnosis. Right heart catheterization and vasoreactivity RHC is required to confirm the diagnosis of PAH and CTEPH, to assess the severity of haemodynamic impairment and to undertake vasoreactivity testing of the pulmonary circulation in selected patients. Cardiac catheterization should be performed after the completion of other investigations.

The availability of molecular genetic diagnosis has opened up a new field for patient care, including genetic counselling for PAH. A positive response to vasoreactivity testing is defined as a reduction of mean PAP e"10 mmHg to reach an absolute value of mean PAP d" $40 \mathrm{mmHg}$ with an increased or unchanged cardiac output. Nitric oxide, Adenosine or Inhaled iloprosts are recommended for performing vasoreactivity..

\section{Professor Sajal Krishna Banerjee \\ Dr. Harisul Hoque \\ Dr. SM Mustafa Zaman \\ Department of Cardiology \\ Bangabandhu Sheikh Mujib Medical University}

\section{Reference:}

2015 ESC/ERS Guidelines for the diagnosis and treatment of pulmonary hypertension 